\title{
Review on the current state of diacylglycerol production using enzymatic approach
}

\begin{abstract}
Enzymatic production of diacylglycerol (DAG)-enriched oil has been investigated extensively due to its health benefits with total annual sales of approximately USD 200 million in Japan since its introduction in the late 1990s till 2009. Enzymatic catalysis had been proven to exhibit improved results with respect to yield, purity, reaction time, and stability in comparison with chemical catalysis. The cost of the enzymes, however, is the main hurdle to the widespread use of enzyme for commercial DAG production. This paper attempts to review and summarize various lipase-mediated technological methods for DAG production. Critical aspects such as process considerations on DAG synthesis, mass transfer limitations as well as kinetic mechanism models developed for each enzymatic approach in DAG synthesis are also presented and discussed. In addition, possible reactor configurations were evaluated, if lipase-assisted DAG production is to be technically and economically feasible at an industrial scale.
\end{abstract}

Keyword: Diacylglycerol; Enzyme lipase; Obesity; Reactor; Kinetic study 